\title{
Spin-wave spectra of a kagome stripe
}

\author{
A. Donkov ${ }^{1}$ and A. V. Chubukov ${ }^{2}$ \\ 1 Max-Planck-Institut für Physik Komplexer Systeme, D-01187 Dresden, Germany \\ 2 Department of Physics, University of Wisconsin, Madison, WI 53706, USA
}

PACS 75.10.Pq - Spin chain models

PACS 75.30.Ds - Spin waves

\begin{abstract}
We study ground state degeneracy and spin-wave excitations in a $1 \mathrm{D}$ version of a Kagome antiferromagnet - a Heisenberg antiferromagnet on a Kagome stripe. We show that for nearest-neighbor interaction, the classical ground state is infinitely degenerate. For any spin configuration from the degenerate set, the classical spin-wave spectrum contains, in addition to Goldstone modes, a branch of zero energy excitations, and a zero mode in another branch. We demonstrate that the interactions beyond nearest neighbors lift the degeneracy, eliminate a zero mode, and give a finite dispersion to formerly zero-energy branch, leaving only Goldstone modes as zero-energy excitations.
\end{abstract}

In the last few years, there has been a revival of interest in the studies of frustrated spin systems. One of the most intriguing aspects of spin frustration is the existence, in many cases, of extra zero modes in the excitation spectrum, in addition to Goldstone modes related to the breaking of a continuous symmetry. These zero modes are often associated with the local degeneracy of a classical ground state of a frustrated system with short-range interactions between nearest-neighbors, and are lifted either by fluctuations, thermal or quantum, or by longer-range interactions which include second, third, etc neighbors [1].

The most prominent example of a system with extra zero modes is a much studied $2 \mathrm{D}$ nearest-neighbor antiferromagnet on a Kagome lattice [2-5]. The Kagome lattice consists of corner-sharing hexagons, and can be obtained from a triangular lattice by removing a quarter of the spins. The classical spectrum of a Kagome antiferromagnet contains the whole branch of zero-energy excitations, associated with the local degeneracy of a classical ground state with respect to rotations of spins belonging to a particular hexagon. Quantum and thermal fluctuations remove the degeneracy and select a particular " $\sqrt{3} \times \sqrt{3}$ " configuration, same as in a triangular antiferromagnet $[3,5,6]$. The same selection, also accompanied by the lifting of zero modes, can be also achieved by adding interactions between next-nearest neighbors [4]. In this later case, the lifting of the degeneracy is a natural consequence of the fact that next-nearest-neighbor interaction connects spins belonging to different hexagons and adds an energy cost to local rotations.

Most of recent work on Kagome-type systems was devoted to $3 \mathrm{D}$ version of a Kagome antiferromagnet, which is an antiferromagnet of the pyrochlore lattice [7]. Less attention was given to an "opposite" $1 \mathrm{D}$ version, which is an antiferromagnet on a Kagome stripe. This is a three-chain structure, consisting of of top/bottom sharing hexagons (Fig. 1b). Like its 2D parent, a Kagome-stripe antiferromagnet can be obtained from a three-chain triangular antiferromagnet by removing $1 / 6$ of the spins (Fig. 1a). The existing analytical $[8,9]$ and numerical $[10,11]$ works focused on $S=1 / 2$ and primarily addressed the issue of a spin-disordered state with gapped spin-triplet excitations, and gapless spin-singlet excitations. Closely related three-spin ladder system have been investigated in [12] by exact diagonalization and DMRG techniques. The studies of Kagome stripes in high magnetic fields have been performed in [13-15].

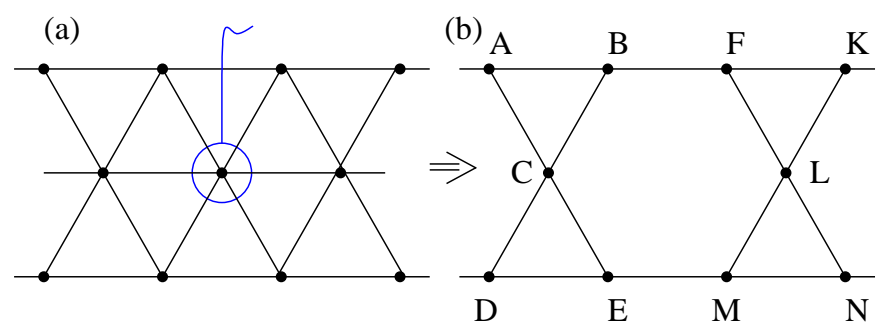

Fig. 1: (a) A triangular three-chain stripe. The Kagome stripe (b) is obtained by taking out the spin in the middle. 
In this communication, we analyze the properties of a Heisenberg Kagome stripe with a large spin $S \gg 1$. We will not discuss the destruction of long-range magnetic order by $1 \mathrm{D}$ fluctuations, which at $T=0$ occurs only at exponentially small energies, but rather focus on the issue of the lifting of the ground-state degeneracy and corresponding zero modes by the interaction beyond nearest neighbors. We show that there are two different ground state degeneracies in a Kagome stripe, besides a conventional global degeneracy which is broken by the long-range order and gives rise to Goldstone modes. One is a truly local degeneracy, which gives rise to a whole branch of zero-energy excitations. Another is an extra global degeneracy with gives rise to an extra zero-mode at a particular momentum $k=0$. We demonstrate that the local degeneracy is lifted by the interactions between spins in the middle chain (formally, third-neighbor interaction), while the extra global degeneracy is lifted by a second-neighbor interaction in the direction perpendicular to the direction of the chains.
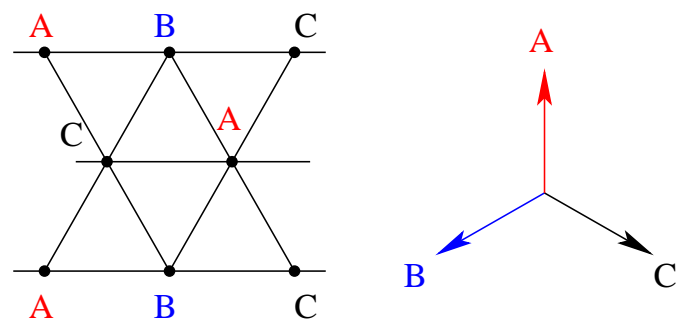

Fig. 2: $120^{\circ}$ spin ordering on a triangular stripe. The directions of the spin quantization axes are labeled by $A, B, C$. The ordering of the whole system is uniquely determined by the $\mathrm{ABC}$ ordering in a given triangle.

The frustrated nature of the Kagome stripe can be most easily understood by comparing it with the triangular stripe in Fig. 1. In both cases, the lowest energy of a particular triangle of spins is achieved by placing the spins $120^{\circ}$ apart. For a triangular stripe with nearestneighbor interaction, the $120^{\circ}$ ordering of a particular triangle uniquely determines the ordering of the full system (see Fig. 21). From this perspective, the triangular stripe of Heisenberg spins is non-frustrated. For a Kagome stripe with nearest-neighbor interaction, the $120^{\circ}$ ordering of a particular triangle does not specify the global order by two reasons. First, the spins D and $\mathrm{E}$ in the triangle DEC, connected to the $\mathrm{ABC}$ triangle by the spin $\mathrm{C}$ in the middle chain (see Fig. 1b), can freely rotate around the quantization axis of the middle-chain spin $\mathrm{C}$ (such rotation is impossible in a triangular antiferromagnet as there the spins $\mathrm{D}$ and $\mathrm{E}$ are also connected to the neighboring spins in the middle chain, whose directions are fixed by the ordering in $\mathrm{ABC}$ triangle. Second, the five spins in the next set of two triangles FKL and LMN, sharing the spin L in the middle chain, are connected to the triangle $\mathrm{ABC}$ only via antiferromagnetic interaction between nearest neighbors $\mathrm{B}$ and $\mathrm{F}$ along the upper chain. This sets the direction (a)
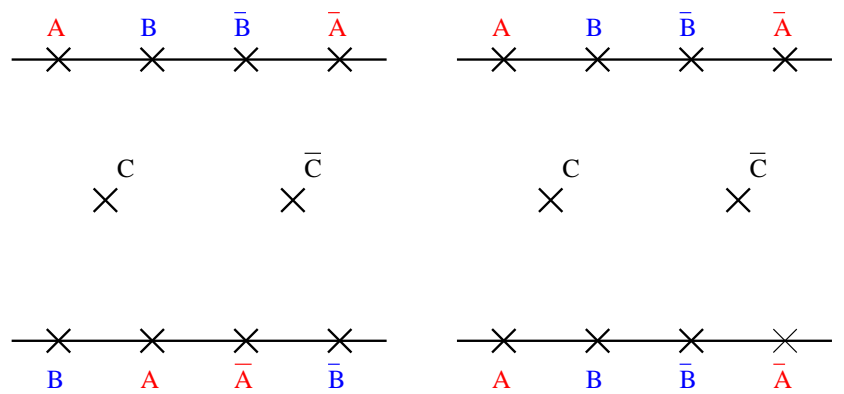

(c)
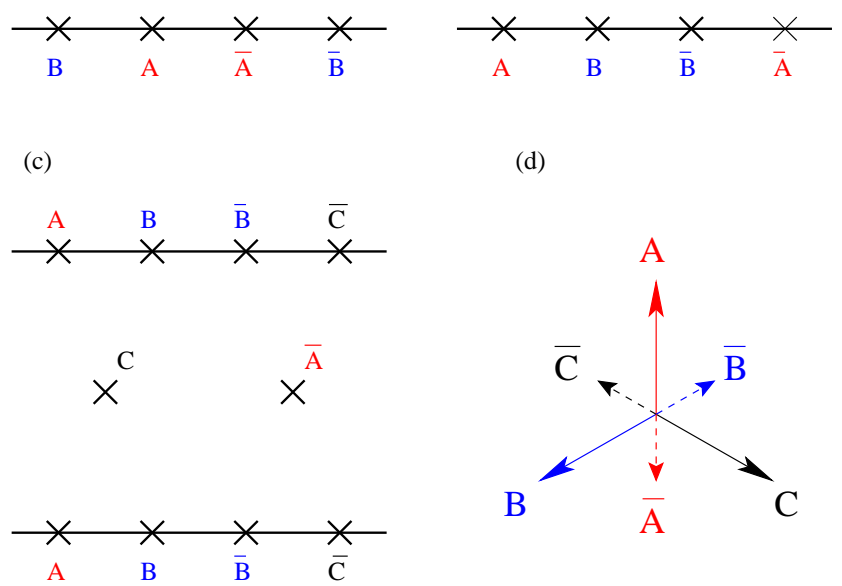

(d)

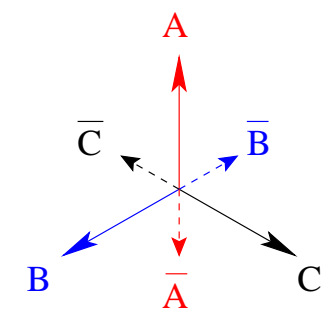

Fig. 3: Different ground state configurations on a Kagome stripe. Each preserves a $120^{\circ}$ ordering within each spin triangle, and antiparallel orientation of the coupled spins from different triangles. The directions $A, B, C$ and their opposites $\bar{A}, \bar{B}, \bar{C}$ are shown in panel (d). The configuration (b) is the $1 \mathrm{D}$ version of the $\sqrt{3} \times \sqrt{3}$ configuration of a $2 \mathrm{D}$ Kagome antiferromagnet. It is stabilized by the interactions between second and third neighbors. For nearest neighbor interaction only, the spins in the configuration $2 \mathrm{~b}$ can be moved without energy cost, as shown in Figs. 4 and 5.

of the spin $\mathrm{F}$, but other four spins can rotate in various ways preserving a $120^{\circ}$ ordering within triangles FKL and LMN. This is clearly a frustrated system.

In Fig. 3 we show three different ground state configurations of a Kagome stripe, each preserves a $120^{\circ}$ ordering within each spin triangle, and antiparallel orientation of the coupled spins from different triangles. For nearestneighbor interaction, the spin-wave spectra of all these configurations are equivalent. The equivalence is broken, however, once we include the interactions between nextnearest neighbors. For definiteness, we focus on the configuration shown in Fig. 3 $\mathrm{b}-$ this is $1 \mathrm{D}$ version of the $\sqrt{3} \times \sqrt{3}$ configuration of a 2D Kagome antiferromagnet. For the configuration of Fig. $3 \mathrm{~b}$, the existence of two different ground state degeneracies can be easily visualized. Indeed, the spins in the middle chain have the same quantization axis (the axis is understood here as a director rather than a vector). One can then rotate the spins in e.g., lower chain around this axis, leaving the spins in the upper chain intact, see Fig. 4. As the spins along the lower chain, belonging to neighboring triangles, interact antiferromagnetically and must remain antiparallel, the 

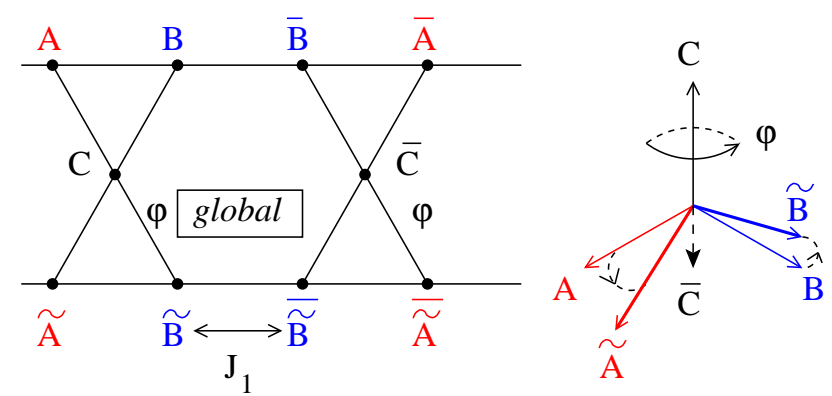

Fig. 4: The "global" degeneracy of the $\sqrt{3} \times \sqrt{3}$ spin configuration. Spins along the lower chain can rotate around the common quantization axis of middle-chain spins $C$ and $\bar{C}$, while the spins along the upper chain remain intact. Since the spins along the lower chain belonging to different sets of top/bottom sharing triangles must remain antiparallel to each other, this rotation simultaneously involves all spins from the lower chain, and is therefore a global rotation. This degeneracy gives rise to a zero mode in the spin-wave spectrum.

rotation must simultaneously involve all spins in the lower chain, and is therefore a global rotation. The degeneracy associated with such rotation is obviously lifted by an interaction $J_{2}$ between a spin in the lower chain and a spin on top of it in the upper chain. A ferromagnetic $J_{2}$ makes the configuration of Fig. $3 \mathrm{~b}$ stable.

Another degeneracy is associated with the rotation of the six spins of a hexagon $B, \bar{B}, \bar{C}, \bar{B}, B, C$ around the common quantization axis of the "corner" spins $A$ and $\bar{A}$, such that $120^{\circ}$ orientations of spins in any triangle is preserved, see Fig. 5. This degeneracy is local as it only involve spins inside a particular hexagon, while the spins and $A$ and $\bar{A}$, through which a given hexagon is connected to other hexagons, remain intact. The local degeneracy is lifted by the interaction $J_{3}$ between the spins in the middle chain, as the angle between these spins obviously changes in the process of a local rotation. An antiferromagnetic $J_{3}$ obviously favors antiparallel orientation of the spins $C$ and $\bar{C}$ and therefore stabilizes the configuration in Fig. $3 \mathrm{~b}$.

To this end, we consider a $J_{1}-J_{2}-J_{3}$ model with Heisenberg interaction within a Kagome stripe $\left(J_{1}\right)$, in between two chains $\left(J_{2}\right)$, and between the spins in the middle chain $\left(J_{3}\right)$ (see Fig $[6$ ). The corresponding Hamiltonian is

$$
H=J_{1} \sum_{<i, i^{\prime}>} \mathbf{S}_{i} \mathbf{S}_{i^{\prime}}-J_{2} \sum_{i} \mathbf{S}_{i, l} \mathbf{S}_{i, u}+J_{3} \sum \mathbf{S}_{i, m} \mathbf{S}_{j, m}
$$

where indices $i, i^{\prime}$ denote the spins belonging to the same set of two top/bottom sharing triangles, $i, j$ denote states belonging to different triangles, and $l, u, m$ denote spins from lower, upper, and middle chains, respectively. We obtain the spin-wave spectrum of this Hamiltonian and show explicitly that the interactions $J_{2}$ and $J_{3}$ lift the zero modes leaving only three Goldstone zero-energy excitations, associated with $\sqrt{3} \times \sqrt{3}$ ordering. These Goldstone modes are related to the breaking of $O(3) \times O(2)$
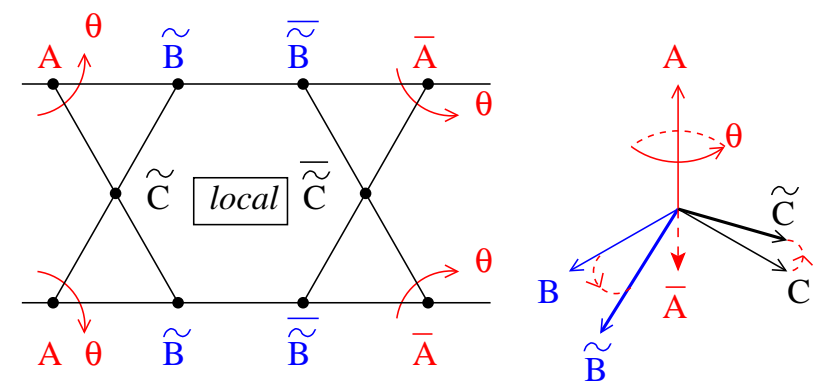

Fig. 5: The "local" degeneracy of the $\sqrt{3} \times \sqrt{3}$ spin configuration. Spins along the hexagon $\tilde{B}, \overline{\tilde{B}}, \overline{\tilde{C}}, \overline{\tilde{B}}, \tilde{B}, \overline{\tilde{C}}$ can rotate around the around the common quantization axis of the "corner" spins $A$ and $\bar{A}$, preserving the $120^{\circ}$ orientations of spins in any triangle. This degeneracy is local as it only involve spins inside a particular hexagon. The local degeneracy gives rise to zero-energy branch of spin-wave excitations.

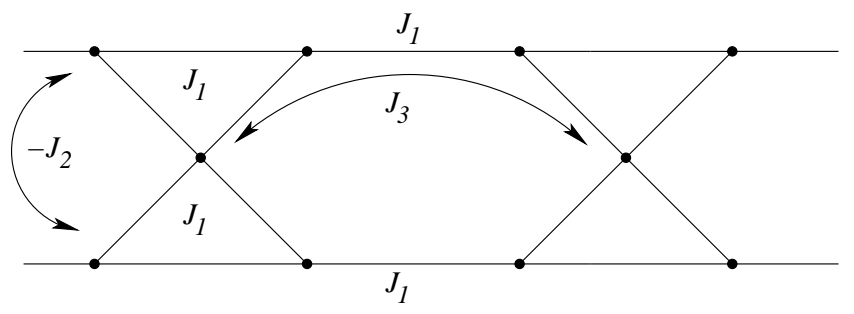

Fig. 6: Kagome stripe with antiferromagnetic nearest neighbor and third-neighbor interactions, $J_{1}$ and $J_{3}$, respectively, and a ferromagnetic second-neighbor exchange interaction $J_{2}$. The Hamiltonian for this model is given by Eq. (1).

symmetry by $\sqrt{3} \times \sqrt{3}$ ordering [17], and are: (i) a homogeneous rotation of $A$ and $B$ spins, in phase with respect to upper and lower chains, and out of phase with respect to $A$ and $B$ directions, (ii) a homogeneous rotation of $A, B$, and $C$ spins - in phase with respect to upper and lower chains, and in phase with respect to $A$ and $B$, and $C$ spins, and (iii) a rotation with $k=\pi / 2$, in phase with respect to upper and lower chains, and in phase with respect to $A$ and $B$ spins, but out of phase with respect to $C$ vs $A$ and $B$ spins.

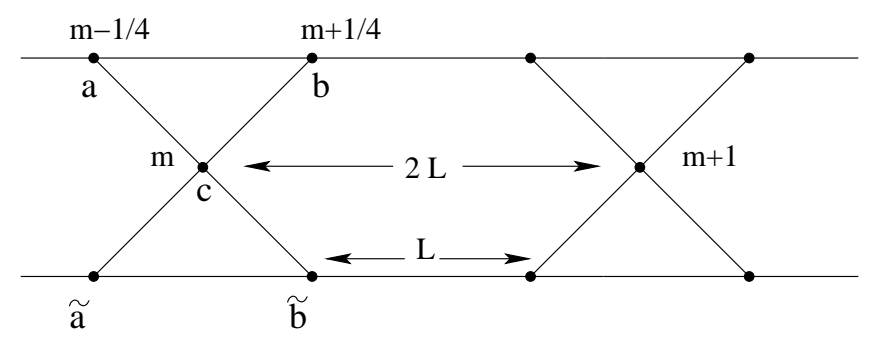

Fig. 7: Notations for the Holstein-Primakoff and Fourier transformations. The distance between neighboring spins along the chains is set to $L=1$.

We follow conventional strategy of the large-S approach: introduce five different bosonic operators for five spins belonging to two top/bottom sharing triangles, use Holstein- 
Primakoff transformation from spin operators to bosons, and diagonalize the quadratic form in boson operators. This is straightforward, but quite cumbersome procedure. We label the five bosons as shown in Fig. 7] set the distance between the sites along the chains to be one, and introduce Fourier components as [16] $a_{m-1 / 4}=$ $\sum_{k} e^{2 i k m} a_{k}, b_{m+1 / 4}=\sum_{k} e^{2 i k m} b_{k}, c_{m}=\sum_{k} e^{2 i k m} c_{k}$, $\tilde{a}_{m-1 / 4}=\sum_{k} e^{2 i k m} \tilde{a}_{k}, \tilde{b}_{m+1 / 4}=\sum_{k} e^{2 i k m} \tilde{b}_{k}$. Substituting into Hamiltonian, we obtain $H=H_{0}+H_{2}+H_{\text {int }}$, where $H_{0}=O\left(S^{2}\right)$ is the classical ground state energy, $H_{2}=O(S)$ describes non-interacting spin-waves, and $H_{\text {int }}=O(1)$, which we neglect below, describes the interaction between spin-waves.

For the spin-wave part, we obtain, explicitly

$$
\begin{gathered}
H_{2}=J_{1} S \sum_{k}\left[2 a_{k}^{\dagger} a_{k}+2 c_{k}^{\dagger} c_{k}+2 b_{k}^{\dagger} b_{k}+2 \tilde{a}_{k}^{\dagger} \tilde{a}_{k}\right. \\
+2 \tilde{b}_{k}^{\dagger} \tilde{b}_{k}+\frac{1}{4}\left(a_{k}^{\dagger} b_{k}+a_{k}^{\dagger} c_{k}+b_{k}^{\dagger} c_{k}\right. \\
\left.+\tilde{a}_{k}^{\dagger} \tilde{b}_{k}+\tilde{a}_{k}^{\dagger} c_{k}+\tilde{b}_{k}^{\dagger} c_{k}+h . c .\right) \\
-\frac{3}{4}\left(a_{k} b_{-k}+a_{k} c_{-k}+b_{k} c_{-k}+\tilde{a}_{k} \tilde{b}_{-k}\right. \\
\left.+\tilde{a}_{k} c_{-k}+\tilde{b}_{k} c_{-k}+h . c .\right) \\
\left.-\left(e^{-i 2 k} b_{k} a_{-k}+e^{-i 2 k} \tilde{b}_{k} \tilde{a}_{-k}+h . c .\right)\right] \\
+J_{2} S \sum_{k}\left[a_{k}^{\dagger} a_{k}+b_{k}^{\dagger} b_{k}+\tilde{a}_{k}^{\dagger} \tilde{a}_{k}+\tilde{b}_{k}^{\dagger} \tilde{b}_{k}\right. \\
\left.-\left(a_{k}^{\dagger} \tilde{a}_{k}+b_{k}^{\dagger} \tilde{b}_{k}+h . c .\right)\right] \\
+2 J_{3} S \sum_{k}\left[c_{k}^{\dagger} c_{k}-\frac{\cos (2 k)}{2}\left(c_{k} c_{-k}+h . c .\right)\right] .
\end{gathered}
$$

Introducing the combinations of operators

$$
a_{1,2}(k)=\frac{1}{\sqrt{2}}\left(a_{k} \pm \tilde{a}_{k}\right), b_{1,2}(k)=\frac{1}{\sqrt{2}}\left(b_{k} \pm \tilde{b}_{k}\right),
$$

we find that the quadratic form is decoupled into a quadratic form which involves $a_{1}(k), b_{1}(k)$, and $c_{k}$ operators (which describe in-phase rotations of the spins in the upper and lower chains), and the quadratic form which involves only $a_{2}(k)$ and $b_{2}(k)$ operators (it describes out-ofphase rotations of the spins in the upper and lower chains). We then have

$$
H_{2}=H_{2 \times 2}+H_{3 \times 3},
$$

where

$$
\begin{aligned}
H_{2 \times 2}=J_{1} S \sum_{k}\left\{2 a_{2}^{\dagger}(k) a_{2}(k)+2 b_{2}^{\dagger}(k) b_{2}(k)\right. \\
+\frac{1}{4}\left(a_{2}^{\dagger}(k) b_{2}(k)+h . c .\right) \\
\left.-\left[\left(\frac{3}{4}+e^{i 2 k}\right) a_{2}(k) b_{2}(-k)+h . c .\right]\right\} \\
+J_{2} S \sum_{k} 2 a_{2}^{\dagger}(k) a_{2}(k)+2 b_{2}^{\dagger}(k) b_{2}(k),
\end{aligned}
$$

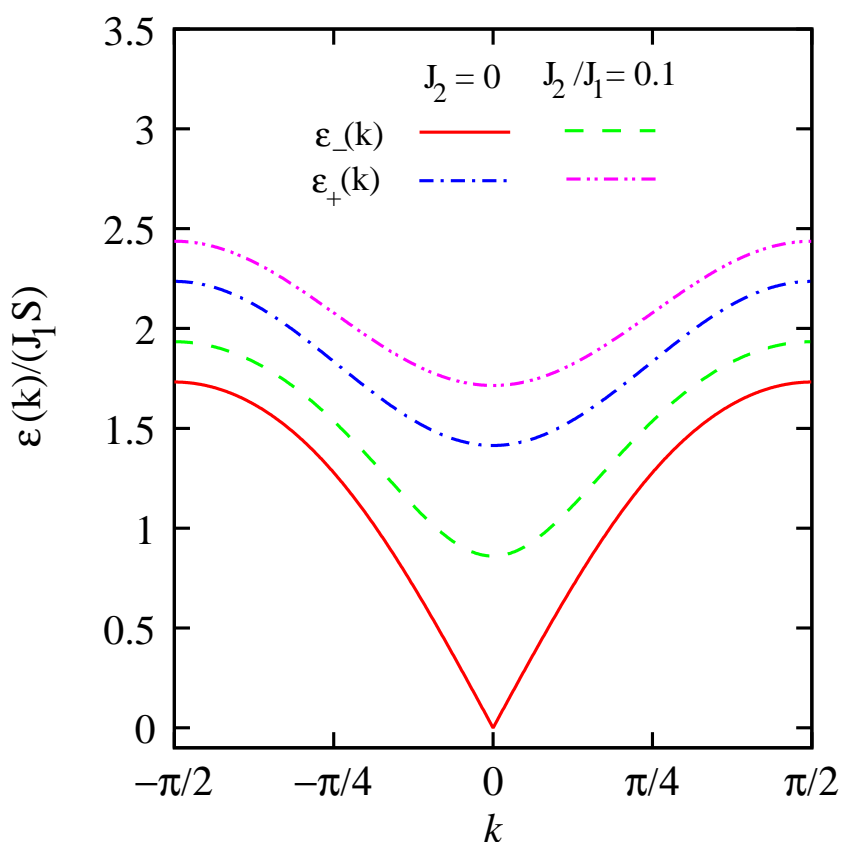

Fig. 8: The spectrum of $H_{2 \times 2}$. The two branches $\epsilon_{ \pm}$are shown for $J_{2}=0$ (full line and dash-doted line), and for $J_{2} / J_{1}=0.1$ (dashed and dashed-two-dotted lines). A finite $J_{2}$ removes the zero mode at $k=0$.

$$
\begin{aligned}
H_{3 \times 3}=J_{1} S \sum_{k}\left\{2 a_{1}^{\dagger}(k) a_{1}(k)+2 b_{1}^{\dagger}(k) b_{1}(k)\right. \\
+\frac{1}{4}\left(a_{1}^{\dagger}(k) b_{1}(k)+h . c .\right) \\
-\left[\left(\frac{3}{4}+e^{i 2 k}\right) a_{1}(k) b_{1}(-k)+h . c .\right] \\
+2 c_{k}^{\dagger} c_{k}+\frac{\sqrt{2}}{4}\left[A_{1}^{\dagger}(k) c_{k}+B_{1}^{\dagger}(k) c_{k}+h . c .\right] \\
\left.-\frac{3 \sqrt{2}}{4}\left[a_{1}(k) c_{-k}+b_{1}(k) c_{-k}+h . c .\right]\right\} \\
+2 J_{3} S \sum_{k}\left[c_{k}^{\dagger} c_{k}-\frac{\cos (2 k)}{2}\left(c_{k} c_{-k}+c_{k}^{\dagger} c_{-k}^{\dagger}\right)\right] .
\end{aligned}
$$

The $H_{2 \times 2}$ part depends on $J_{1}$ and $J_{2}$ but not on $J_{3}$. Like we said, it describes out-of-phase rotations of the spins in the upper and lower chains. Such rotations are not Goldstone modes, and generally the two spin-wave branches of $H_{2 \times 2}$ must be gapped. This, however, happens only at a finite $J_{2}$; for only neatest-neighbor interaction, out-ofphase rotations give rise to an extra zero mode (see Fig. 4).

The Hamiltonian $\mathrm{H}_{2 \times 2}$ can be diagonalized analytically. For this, we note that for a generic Hamiltonian

$$
\begin{aligned}
H_{4 \times 4} & =\sum_{k} C_{1}(k)\left(A_{k}^{\dagger} A_{k}+B_{k}^{\dagger} B_{k}\right)+C_{2}(k)\left(A_{k}^{\dagger} B_{k}\right. \\
& \left.+B_{k}^{\dagger} A_{k}\right)+\left(C_{3}(k) A_{k} B_{-k}+C_{3}^{*}(k) A_{k}^{\dagger} B_{-k}^{\dagger}\right)
\end{aligned}
$$

with real $C_{1}, C_{2}$ and complex $C_{3}$, the excitation spectrum 
is

$\varepsilon_{ \pm}^{2}(k)=\left[\sqrt{C_{1}^{2}(k)-\left(\operatorname{Im} C_{3}(k)\right)^{2}} \pm C_{2}(k)\right]^{2}-\left(\operatorname{Re} C_{3}(k)\right)^{2}$.

In our case, we have $C_{1}(k)=2 S\left(J_{1}+J_{2}\right), \quad C_{2}(k)=$ $J_{1} S / 4, \quad C_{3}(k)=-J_{1} S\left(\frac{3}{4}+e^{2 i k}\right)$, such that $\operatorname{Re} C_{3}(k)=$ $J_{1} S(-3 / 4-\cos (2 k)), \quad \operatorname{Im} C_{3}(k)=-J_{1} S \sin (2 k)$. Substituting into (6), we obtain two branches of excitations with the dispersion:

$$
\begin{aligned}
\varepsilon_{ \pm}(k) & =J_{1} S\left\{\frac{5}{2}-\frac{3 \cos (2 k)}{2}+\left(\frac{8 J_{2}}{J_{1}}+\frac{4 J_{2}^{2}}{J_{1}^{2}}\right)\right. \\
\pm & \left.\frac{1}{2}\left[\frac{7}{2}+\frac{\cos (4 k)}{2}+\left(\frac{8 J_{2}}{J_{1}}+\frac{4 J_{2}^{2}}{J_{1}^{2}}\right)\right]^{1 / 2}\right\}^{1 / 2} .
\end{aligned}
$$

The spectrum is shown in Fig 8 for $J_{2}=0$ and $J_{2} / J_{1}=$ 0.1 . In the absence of $J_{2}$, the dispersion $\varepsilon_{-}(k)$ has a zero mode at $k=0$. At a nonzero $J_{2}$, the zero mode is lifted, and the dispersion acquires a finite gap, as we anticipated. The other dispersion branch, $\varepsilon_{+}(k)$, is gapped already at $J_{2}=0$, and does not change significantly with $J_{2}$.

The diagonalization of the $H_{3 \times 3}$ part of the Hamiltonian is more involved as this Hamiltonian contains three operators with complex coefficients. The diagonalization amounts to solving 6 by 6 matrix which we did numerically. The results for $J_{3}=0$ and $J_{3}=0.3 J_{1}$ are plotted in Fig. 9 There are three branches of magnon dispersion. They describe in phase and out-of-phase rotations between $A, B$, and $C$ spins - in all cases the rotations are symmetric with respect to spins in the upper and lower chains. The three Goldstone modes are among these excitations.

For $J_{3}=0$, one of the branches is gapped, another is linear in $k$ near $k=0$, and the third one is exactly zero for all $k$-points. This is the consequence of the local degeneracy. All three branches can be easily obtained analytically as at $J_{3}=0$, the zero-energy branch decouples from the other two branches, which are the same as $\varepsilon_{ \pm}(k)$ for $J_{2}=0$. At a finite $J_{3}$, the local degeneracy is lifted, and the former zero-energy branch acquires a "sin $2 k$ "-like dispersion with finite energy at a generic $k$, and Goldstone points at $k=0$ and $k= \pm \pi / 2$. The linear in $k$ branch remains gapless and its velocity is only slightly affected by $J_{3}$.

The existence of three Goldstone modes at a finite $J_{3}$ agrees with what one should expect on general grounds. Furthermore, the Goldstone modes can be obtained analytically. For $k=0$ and $k=\pi / 2$, the out-of-phase mode $p_{-}=\left(a_{1}-b_{1}\right) / \sqrt{2}$ is decoupled from the modes $p_{+}=\left(a_{1}+b_{1}\right) / \sqrt{2}$ and $c$. At $\mathrm{k}=0$, the energy of $p_{-}$ mode is zero (Goldstone), at $k=\pi / 2$, it is $J_{1} S \sqrt{3}$, independent on $J_{3}$. For the remaining two coupled excitations, $p_{+}$and $c$, the Hamiltonian can be diagonalized in the same spirit as $H_{2 \times 2}$. At $k=0$, we obtain one solution at zero energy (Goldstone), and one at energy $\bar{\varepsilon}_{+}(k=0)=J_{1} S \sqrt{2+8 J_{3} / J_{1}}$, at $k=\pi / 2$, one solution has zero energy (Goldstone), another is at energy

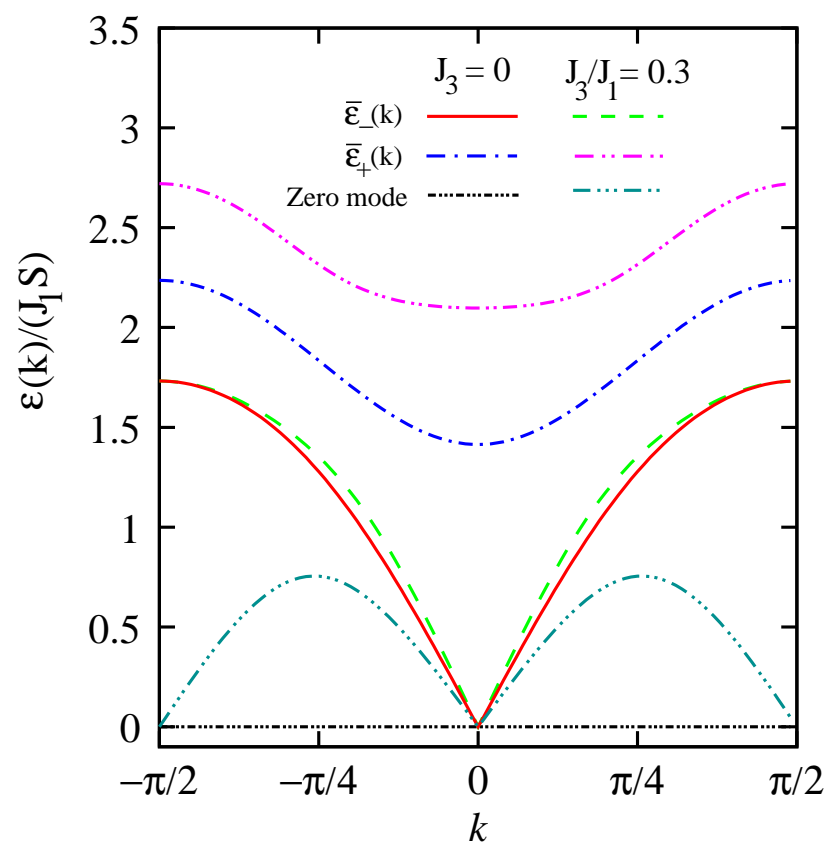

Fig. 9: The spectrum of $H_{3 \times 3}$. Without $J_{3}$, the dispersion has one linear in $k$ (Goldstone) branch, and one branch of zero energy excitations. When $J_{3}$ is finite, the former zero-energy branch acquires a dispersion and yields two Goldstone modes at $k=0$ and $k=\pi / 2$. The third branch is gapped with and without $J_{3}$.

$\bar{\varepsilon}_{+}(\pi / 2)=J_{1} S \sqrt{5+8 J_{3} / J_{1}}$. The combinations of $p_{+}$and $c$ for the Goldstone modes are $2 p_{+}+c$ at $k=0$ and $p_{+}-c$ at $k=\pi / 2$.

In summary, in this paper, we considered a Heisenberg model on a Kagome stripe. We showed that for nearest-neighbor interaction, the quasiclassical excitation spectrum contains two special features associated with the extra degeneracies of a classical ground state, a zeroenergy branch of excitations associated with a local degeneracy, and a single zero mode associated with an additional global degeneracy. We demonstrated that the zero mode and zero-energy branch are removed by second- and thirdneighbor interactions, respectively. We obtained the full quasiclassical spin-wave spectrum for a model with the interaction between first, second, and third neighbors, and showed that it contains three Goldstone modes, precisely as it should be for a ground state which breaks $O(3) \times O(2)$ symmetry.

A.V.C. acknowledge the support from nsf-dmr 0604406 , and the hospitality of the TU Braunschweig and MPIPKS in Dresden during the completion of this work.

\section{REFERENCES}

[1] Shender E. F., Sov. Phys. - JeTP, 56 (1982) 178; HenLey C. L., Phys. Rev. Lett., 62 (1989) 2056; Korshunov S. E., Phys. Uspekhi, 49 (2006) 225.

[2] Zeng C. and Elser V., Phys. Rev. B, 42 (1990) 8436.

[3] Sachdev S., Phys. Rev. B, 45 (1992) 12377. 
[4] Harris A. B., Kallin C. and Berlinsky A. J., Phys. Rev. B, 45 (1992) 2899.

[5] Chubukov A., Phys. Rev. Lett., 69 (1992) 832; Chubukov A., J. Appl. Phys., 73 (1993) 5639.

[6] Zhitomirsky M. E., Phys. Rev. Lett., 88 (2002) 057204.

[7] see e.g., Tchernyshyov O., Starykh O. A., Moessner R. and Abanov A. G., Phys. Rev. B, 68 (2003) 144422, and references therein.

[8] Azaria P., Hooley C., Lecheminant P., Lhuillier C. and Tsvelik A. M., Phys. Rev. Lett., 81 (1998) 1694.

[9] Azaria P., Hooley C., Lecheminant P., Lhulllier C. and Tsvelik A. M., Phys. Rev. Lett., 85 (2000).

[10] Pati S. K. and Singh R. R. P., Phys. Rev. B, 60 (1999) 7695.

[11] White S. R. and Singh R. R. P., Phys. Rev. Lett., 85 (2000) .

[12] Waldtmann C., Kreutzmann H., Schollwöck U., Maisinger K. and Everts H. U., Phys. Rev. B, 62 (2000) .

[13] Derzhko O. and Richter J., Eur. Phys. Jour. B, 52 (2006) 23.

[14] Schnack J., Schmidt H. J., Honecker A., SchulenBurg J. and Richter J., J. Phys.: Conf. Series, 51 (2006) 43.

[15] Schmidt H.-J. U., Richter J. and Moessner R., J. Phys. A, 39 (2006) 10673.

[16] Khveshchenko D. V. and Chubukov A. V., Sov. Phys. - JETP, 66 (1987) 1088.

[17] Dombre T. and Read N., Phys. Rev. B, 39 (1989) 6797. 